\title{
Long-Term Fluences of Energetic Particles in the Heliosphere
}

\author{
R. A. Mewaldt ${ }^{1}$, G. M. Mason ${ }^{2}$, G. Gloeckler ${ }^{2}$, E. R. Christian ${ }^{3}$, C. M. S. Cohen ${ }^{1}$, \\ A. C. Cummings ${ }^{1}$, A. J. Davis ${ }^{1}$, J. R. Dwyer ${ }^{4}$, R. E. Gold ${ }^{5}$, S. M. Krimigis ${ }^{5}$, \\ R. A. Leske ${ }^{1}$, J. E. Mazur ${ }^{6}$, E. C. Stone ${ }^{1}$, T. T. von Rosenvinge ${ }^{3}$, \\ M. E. Wiedenbeck ${ }^{7}$, and T. H. Zurbuchen ${ }^{8}$ \\ ${ }^{1}$ California Institute of Technology, Pasadena, CA 91125 USA \\ ${ }^{2}$ University of Maryland, College Park, MD 20742 USA \\ ${ }^{3}$ NASA/Goddard Space Flight Center, Greenbelt, MD 20771 USA \\ ${ }^{4}$ Florida Institute of Technology, Melbourne, FL 32901 USA \\ ${ }^{5}$ Johns Hopkins University/Applied Physics Laboratory, Laurel, MD 20723 USA \\ ${ }^{6}$ The Aerospace Corporation, El Segundo, CA, 90009 USA \\ ${ }^{7}$ Jet Propulsion Laboratory, Pasadena, CA 91009 USA \\ ${ }^{8}$ University of Michigan, Ann Arbor, MI 48109 USA
}

\begin{abstract}
We report energy spectra of $\mathrm{He}, \mathrm{O}$, and Fe nuclei, extending from $\sim 0.3 \mathrm{keV} /$ nucleon to $\sim 300$ $\mathrm{MeV} /$ nucleon, integrated over the period from the Fall of 1997 to mid-2000. These fluence measurements were made at 1 AU using data from the SWICS, ULEIS, SIS, and CRIS instruments on ACE, and include contributions from fast and slow solar wind, coronal mass ejections, pickup ions, impulsive and gradual solar particle events, acceleration in corotating interaction regions and other interplanetary shocks, and anomalous and galactic cosmic rays. Fluence measurements of six additional species are presented in the energy region from $\sim 0.04$ to $\sim 100 \mathrm{MeV} /$ nucleon. We discuss the relative contributions of the various particle components, and comment on the shape and time dependence of the measured energy spectra.
\end{abstract}

\section{INTRODUCTION}

The various components of energetic particles observed in the heliosphere include rather steady sources such as the solar wind, pickup ions, and anomalous and galactic cosmic rays, as well as transient sources such as solar energetic particle events and interplanetary shocks. At the lowest energies, the typical scale of variations in the velocity, intensity, and composition of the solar wind is a factor of $\sim 2$, on time scales that range from hours to days to years. At the highest energies, anomalous and galactic cosmic rays vary in intensity over the solar cycle, but are essentially constant in composition. At intermediate energies ranging from the $\sim 30 \mathrm{keV} /$ nucleon to $\sim 30$ $\mathrm{MeV} /$ nucleon, the intensity, spectra, and composition of heliospheric particles are all observed to be highly variable (particularly at solar maximum), and apparently originate from a number of separate sources and acceleration processes. It is therefore of interest to integrate these highly variable components over an extended time period to obtain a direct measure of the longer-term fluence of energetic particles that originate on the Sun, in the heliosphere, and in the Galaxy.

The experiments on ACE are ideal for this kind of measurement, because they measure composition and energy spectra continuously from solar wind energies of $\sim 1 \mathrm{keV} /$ nucleon to cosmic ray energies of a few hundred $\mathrm{MeV} /$ nucleon [1]. In particular, ACE is able to explore the composition and energy spectra of ions in the relatively unknown suprathermal region from a few $\mathrm{keV} /$ nucleon to a few hundred $\mathrm{keV} /$ nucleon.

In this paper we report preliminary measurements of the long-term fluence of ions over six decades in energy/nucleon during a 33-month time period that includes both solar-minimum and solar-maximum conditions. The results of these measurements are of interest to understanding processes of particle acceleration and transport on the Sun and in the heliosphere, and to understanding the long-term contributions of energetic-particles to the lunar soil, meteorites, and planetary atmospheres. In a separate paper [2], we compare the observed fluences of solar wind and suprathermal nuclei with observations of energetic ions implanted in the lunar soil. 


\section{INSTRUMENTATION AND DATA ANALYSIS}

The measurements reported here were obtained from the SWICS [3], ULEIS [4], SIS [5], and CRIS [6] instruments on ACE (see Table 1). Energetic particle fluxes from ULEIS, SIS and CRIS were accumulated over a 33-month time period extending from 1997:280 to $2000: 184$. Fluence data were obtained by summing hourly-average fluxes within a large number of separate energy intervals, taking into account the measured instrument live times and geometry factors. (Such hourly-average fluxes are a routine product of ACE Level-2 data processing, and are available at http://www.srl.caltech.edu/ACE/ASC/). Note that this period starts with solar-minimum conditions in 1997 and ends with solar-maximum conditions in 2000.

Table 1: Sources of Fluence Data

$\begin{array}{ll}\text { SWICS } & 0.0005 \text { to } 0.030 \mathrm{MeV} / \mathrm{nuc} \\ \text { ULEIS } & 0.040 \text { to } \sim 5 \mathrm{MeV} / \mathrm{nuc} \\ \text { SIS } & \sim 8 \text { to } \sim 100 \mathrm{MeV} / \text { nuc } \\ \text { CRIS } & \sim 80 \text { to } \sim 300 \mathrm{MeV} / \mathrm{nuc}\end{array}$

The SWICS data for this preliminary report were summed over an 11-month period extending from January through November in 1999. These 11-month fluences were multiplied by a factor of 3 to correspond to the 33-month period used by the higher-energy instruments. Because the solar wind is less variable than higher-energy solar and interplanetary components, this 1999 period should provide a reasonable representation of the longer time period.

As expected, this first comparison of absolute fluence measurements from four instruments led to renormalization of some of the measurements. The fluence spectra from SIS and CRIS were found to be in agreement to within $10 \%$ where they overlapped, and were left unchanged. During the largest SEP events the ULEIS fluxes were somewhat low due to reduced detection efficiency in periods with intense fluxes. The correction factors for ULEIS during these intense periods were obtained by comparison with fluxes measured using the STEP sensor on Wind [7] and the ACE/SIS instrument; these latter two sensors are less susceptible to saturation than ULEIS. The correction to ULEIS fluences for $\mathrm{He}$ and $\mathrm{CNO}$ was a factor of 1.5 below $1.5 \mathrm{MeV} /$ nucleon, and a factor of 2.2 at higher energies, while no corrections were made for species from $\mathrm{Ne}$ to $\mathrm{Fe}$.

After applying small energy-dependent efficiency corrections, the ACE/SWICS oxygen abundance (based on previously un-calibrated matrix-rate data [3]) was normalized to the solar-wind composition measured by Ulysses/SWICS [8], which also brings it into reasonable agreement with the composition measured by ULEIS. These issues of absolute normalization will be examined in more detail in the future.

\section{FLUENCE MEASUREMENTS}

Measured fluences of $\mathrm{He}, \mathrm{O}$, and $\mathrm{Fe}$ extending from solar wind to cosmic ray energies are shown in Figure 1. All three species clearly have a common spectral shape. The peak at $\sim 0.8 \mathrm{keV} /$ nucleon in all three spectra corresponds to the slow-speed solar wind with a mean velocity of $\sim 400 \mathrm{~km} / \mathrm{sec}$. The contribution of occasional higher-speed streams, sometimes including velocities as high as $1000 \mathrm{~km} / \mathrm{sec}$ can also be seen. Including the intrinsic thermal speed of the solar wind, it appears from Figure 1 that the solar wind distribution extends to between 5 and $10 \mathrm{keV} /$ nucleon before there is a change in slope. Beyond this is a long, suprathermal tail extending with a power- law slope of -2 to $\sim 10$ $\mathrm{MeV} /$ nucleon. Near $\sim 10 \mathrm{MeV} /$ nucleon all spectra exhibit a gradual "knee" and briefly steepen. Above $\sim 100 \mathrm{MeV} /$ nucleon, the modulated fluence of galactic cosmic rays (GCRs) begins to dominate, continuing on for many more decades in energy (see, e.g., [9]).

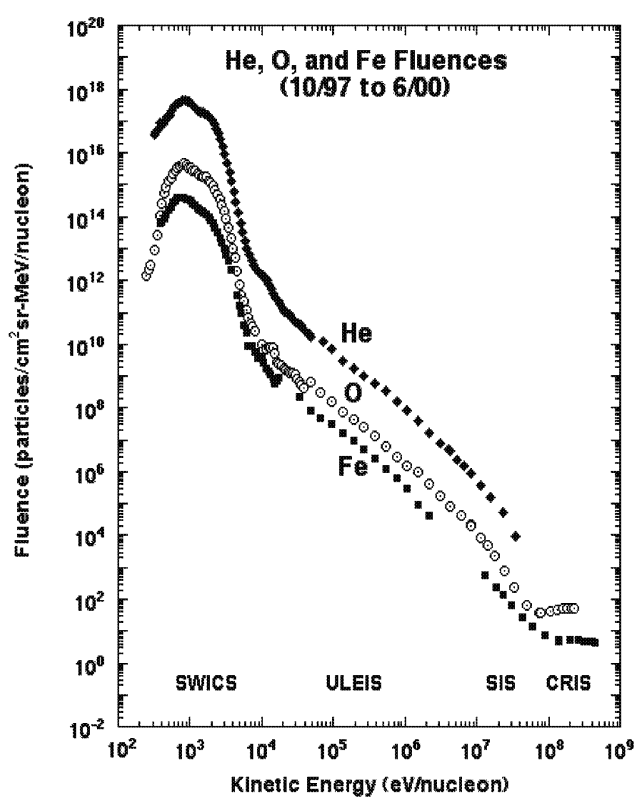

Figure 1: Fluences of $\mathrm{He}, \mathrm{O}$, and $\mathrm{Fe}$ nuclei measured over the period from $9 / 1997$ to $6 / 2000$ by the SWICS, ULEIS, SIS, and CRIS instruments on ACE. The SWICS fluences were measured over the first eleven months of 1999 and then multiplied by $x 3$. The He fluence includes both doubly and singly-charged He. 
In the intermediate region from $\sim 30 \mathrm{keV} /$ nucleon to $\sim 30 \mathrm{MeV} /$ nucleon the fluence spectra are a superposition of many separate "events". Some examples of these events are illustrated for oxygen in Figure 2. At energies from $\sim 3$ to $30 \mathrm{MeV} /$ nucleon most of the fluence comes from the largest solar particle events that occur a few times a year during solar maximum (e.g., the 11/97 and 4/98 events). A time-intensity plot of high-energy SEP events observed by ACE appears in Figure 1 of Cohen et al. [10]; Figure 3 illustrates how these events contribute to the $10 \mathrm{MeV} /$ nucleon fluence as a function of time. Note that over this 3-year period anomalous cosmic rays make only a very small contribution to the fluence at $1 \mathrm{AU}$ - the contributions of large SEP events are considerably greater.

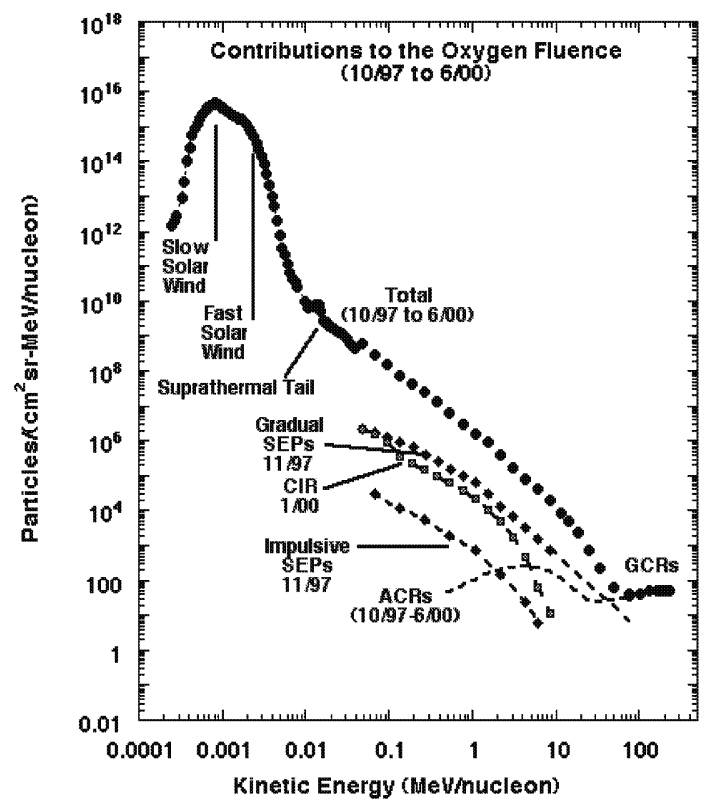

Figure 2: Illustration of some of the contributions to the oxygen fluence, including examples of impulsive and gradual SEP events, CIR-accelerated particles, and anomalous and galactic cosmic rays.

In the energy range from $\sim 0.1$ to $1 \mathrm{MeV} /$ nucleon, there are important contributions from impulsive solar flares and from particles accelerated in corotating interaction regions (CIRs). Although these events are generally much smaller in size than gradual SEP events, the impulsive events, in particular, occur much more frequently. At $\sim 0.1 \mathrm{MeV} /$ nucleon there are no large individual events that dominate - rather, there appear to be similar contributions from as many as 100 separate events of various kinds (see Figure 4).
At even lower energies $(\sim 10 \mathrm{keV} /$ nucleon to $\sim 50$ $\mathrm{keV} /$ nucleon, measurements with SWICS/ACE have shown that suprathermal tails on the solar wind are continuously present [11], but the origin of these tails is presently a subject of investigation (see discussion below). At $\sim 1 \mathrm{keV} /$ nucleon the intensity of singlycharged interstellar and inner-source pickup ions is

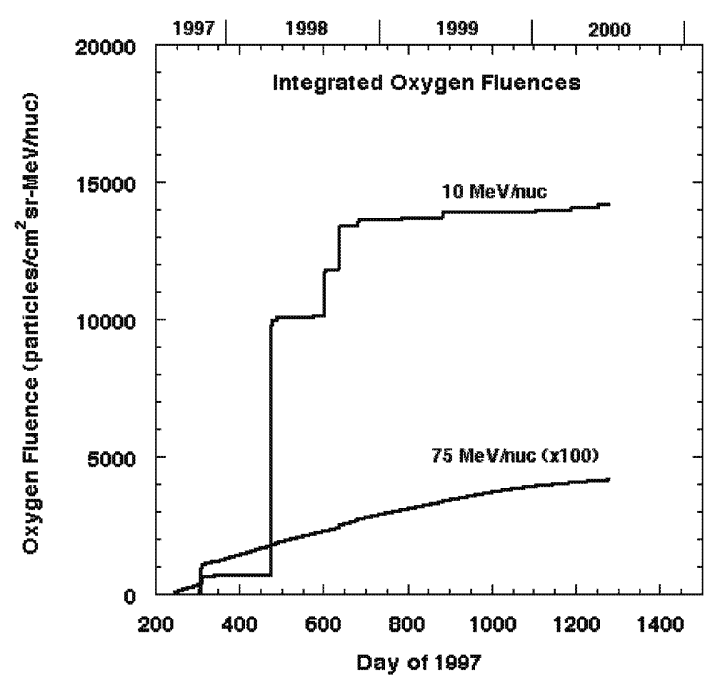

Figure 3: Time history of the integrated oxygen fluences at 10 and at $75 \mathrm{MeV} /$ nucleon measured by SIS. In this case 27-day average fluxes were integrated. At $10 \mathrm{MeV} /$ nucleon a few large SEP events contribute more than the continuous anomalous cosmic ray intensity. At $75 \mathrm{MeV} /$ nucleon the steady, contribution of galactic cosmic rays is apparent.

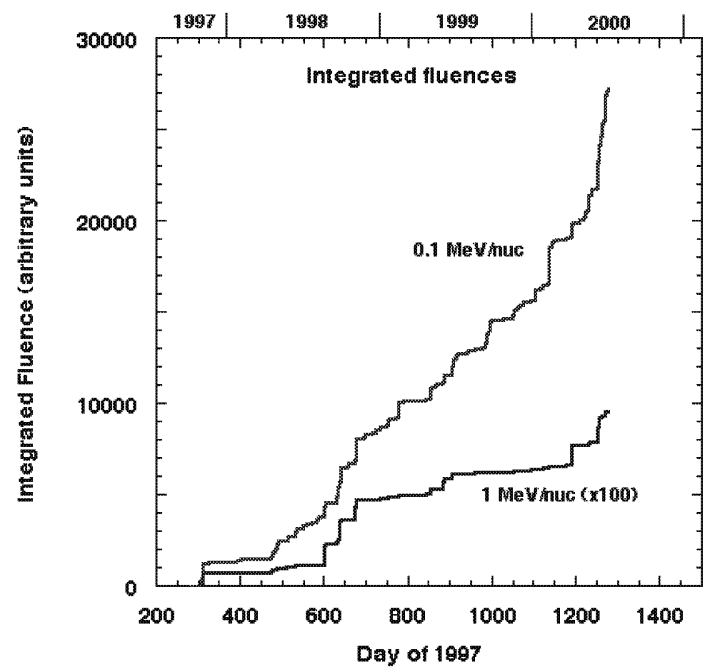

Figure 4: Time history of integrated daily oxygen fluences at 0.1 and $1 \mathrm{MeV} /$ nucleon measured by ULEIS. Note that a large number of individual events contribute. 
estimated to be several orders of magnitude lower than the solar-wind intensity (see Figure 9 of [11]). Using ULEIS and SIS data, fluence spectra have been constructed for a total of 9 species, extending from $\sim 0.04$ to $\sim 100 \mathrm{MeV} /$ nucleon. It is rather remarkable that all of these species show the same $\mathrm{E}^{-2}$ power-law spectral shapes from $\sim 0.04 \mathrm{MeV} /$ nucleon to $\sim 10$ $\mathrm{MeV} /$ nucleon, as demonstrated in Figure 5. Figure 6 compares the relative abundances at three representative energies. The three compositions appear to be very similar; there is no obvious evidence for contributions from sources with an unusual composition. Statistical uncertainties are negligible in Figure 6, but because data from two of the instruments was re-normalized, it is possible that there could be systematic uncertainties of as much as a factor of $\sim 2$.

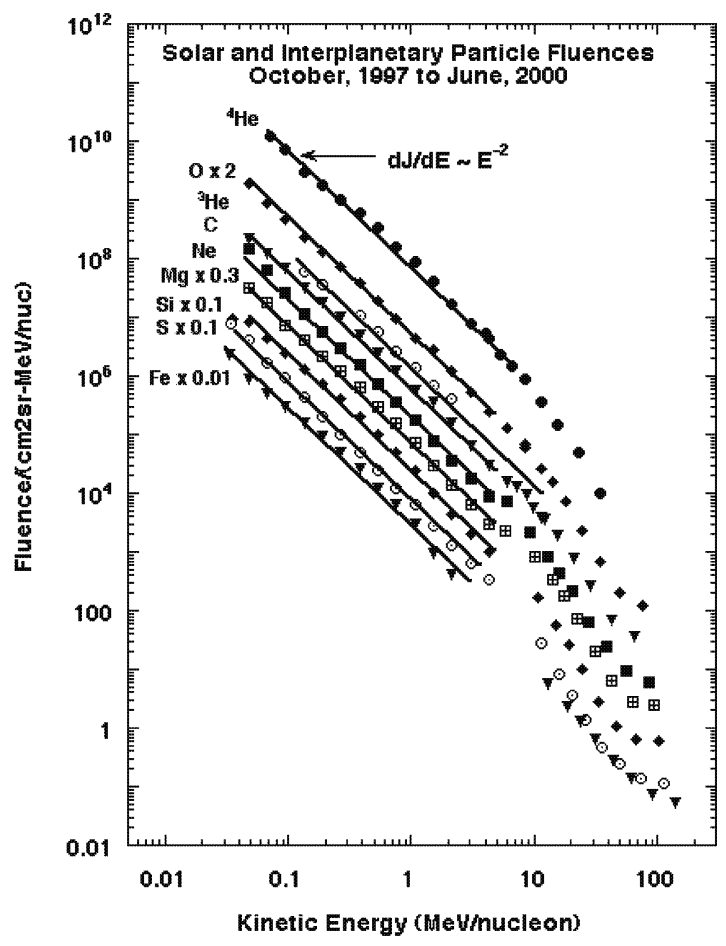

Figure 5: Solar and interplanetary fluences of nine species measured by ULEIS and SIS. It is surprising that all species have a common, $\mathrm{E}^{-2}$ power-law spectrum over a large energy range. The $\mathrm{E}^{-2}$ spectra were fit by eye.

\section{DISCUSSION}

It is somewhat surprising that the integrated fluences of ions from such a variable mix of sources show such a high degree of organization. All species are found to have a common spectral shape. In particular, it is remarkable that a common power-law slope applies to the region extending from $\sim 10$ $\mathrm{keV} /$ nucleon, where the suprathermal tails on the solar wind dominate, to $\sim 10 \mathrm{MeV} /$ nucleon, where large, gradual SEP events contribute most of the fluence.

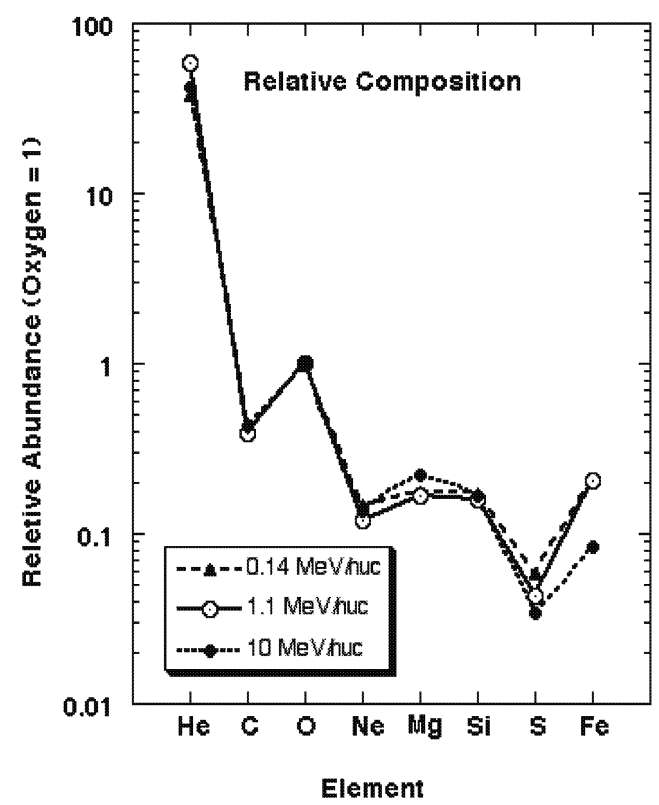

Figure 6: Comparison of the compositions measured at three energies, all normalized to oxygen $=1$.

The origin of the suprathermal solar-wind tails remains a mystery. Although earlier studies observed similar tails in association with CIRs [12] or other interplanetary shocks, ACE studies of quiet time periods have shown that these tails are continuously present in the in-ecliptic solar wind, even when there are no shocks present [11]. The fact that tails are observed on the distributions of interstellar pickup ions as well as on solar-wind species argues that they must originate in interplanetary space and not on the Sun [11]. Fisk et al. [13] suggest that they are due to statistical acceleration by transit-time damping, but there is not as yet a consensus on their origin.

There are also questions as to the extent of the tails. In Figure 1 it is seen that the power-law portion of the spectrum extends continuously from $\sim 10 \mathrm{keV} /$ nucleon to $\sim 10 \mathrm{MeV} /$ nucleon, suggesting that all of these ions have a common origin. However, from Figures 3 and 4 it is appears that while a continuous process (or processes) might explain the bulk of the particles observed at $\sim 0.1 \mathrm{MeV} /$ nucleon, at $1 \mathrm{MeV} /$ nucleon and at $10 \mathrm{MeV} /$ nucleon isolated, individual events 
contribute most of the integrated fluence. It is clear that the origin of these tails will be a source of lively debate over the coming years.

Composition measurements provide some clues to the origin of the particles. The measured ${ }^{3} \mathrm{He} /{ }^{4} \mathrm{He}$ ratio above $100 \mathrm{keV} /$ nucleon is $\sim 0.01-0.02$, a factor of more than 20 greater than the corresponding ratio in the solar wind. While this suggests that impulsive ${ }^{3} \mathrm{He}-$ rich solar flare events make an appreciable contribution to this energy region, ${ }^{3} \mathrm{He}$ is also overabundant in many gradual SEP events [14, 15], and it is likely that the ${ }^{3} \mathrm{He}$ fluence is due in part to the re-acceleration of remnant impulsive-flare material by interplanetary shocks $[15,16]$.

The time period covered by this study ended just before the "Bastille Day" event on July 14, 2000, the largest SEP event during this solar cycle. It is reasonable to ask to what extent inclusion of this event would modify the fluences in Figure 1. Using spectra compiled by Tylka et al. (personal communication, 2001) from a number of spacecraft including ACE and Wind, we find that the Bastille Day event contributes only a small fraction $(<10 \%)$ of the 33-month fluences below $0.1 \mathrm{MeV} /$ nucleon. However, over the energy range from $\sim 2-3$ to $\sim 20-30 \mathrm{MeV} /$ nucleon (depending somewhat on species) this single event contributes more than the 33 months combined. The greatest relative contribution is to $\mathrm{He}$, since this event was enriched in He relative to heavier ions. The addition of this contribution does not significantly alter the $\mathrm{E}^{-2}$ character of the $\mathrm{O}$ and $\mathrm{Fe}$ fluences in Figures 1 and 5 , but it does flatten the He spectrum somewhat. There are also other large events after July 2000 that would make significant contributions.

There are several similarities between the heliospheric spectra and the spectra of higher-energy galactic cosmic rays. Common spectral shapes are observed from $\sim 10 \mathrm{keV} /$ nucleon to $\sim 10 \mathrm{MeV} /$ nucleon, even though the individual events contributing to these spectra often have non-power-law spectra, with abundance ratios such as $\mathrm{Fe} / \mathrm{O}$ that vary by a factor ranging from 0.1 to 10 .

Similarly, it is possible that galactic cosmic rays also include contributions from several different sources that may have differing spectra and composition, yet the abundant GCR species from $\sim 10^{9}$ to $\sim 10^{13} \mathrm{eV} /$ nucleon appear to be consistent with a single common source spectrum, in this case somewhat steeper than -2 . Note that in both of these cases the power-law regions end with a "knee", followed at higher energy by an "ankle". Although it is believed that shock-acceleration processes make the dominant contributions to the GCR spectra from $10^{9}$ to $10^{14} \mathrm{eV} /$ nucleon, the situation is presently less clear for the heliospheric spectra from $10^{4}$ to $10^{7} \mathrm{eV} /$ nucleon.

\section{SUMMARY}

Continuous solar-wind, solar-particle, heliospheric, and cosmic-ray fluences have been measured from $\sim 0.3$ $\mathrm{keV} /$ nucleon to $\sim 300 \mathrm{MeV} /$ nucleon. Both solar minimum and solar maximum conditions were included, and we suggest that these spectra provide a good first approximation to the spectral shapes that would be obtained if such measurements were extended over an entire solar cycle. All species are found to have a common spectral shape. The solar wind (including both slow and high-speed wind) dominates the energy range up to $\sim 8 \mathrm{keV} /$ nucleon. In the energy range from $\sim 10 \mathrm{keV} /$ nucleon to $\sim 10 \mathrm{MeV} /$ nucleon as many as 100 or more separate events somehow combine to produce $\mathrm{E}^{-2}$ power-law spectra that are common to all of the nine species measured, including ${ }^{3} \mathrm{He}$.

In the decade from $\sim 5$ to $\sim 50 \mathrm{MeV} /$ nucleon intense, gradual SEP events make the largest contribution, while at higher energies galactic cosmic rays take over. It is not yet known what processes make the largest contributions to the intermediate region from $\sim 10$ $\mathrm{keV} /$ nucleon to $\sim 5 \mathrm{MeV} /$ nucleon. The possibilities include impulsive SEP events, CIR events, or other, as yet unidentified processes that occur continuously on the Sun or in the inner heliosphere. We intend to address this question in the near future.

These are the first spectral measurements to extend continuously from solar-wind to cosmic-ray energies. Given the highly variable composition and intensity of the contributing events, the overall similarity of these fluence spectra is surprising.

\section{References}

1. Stone, E. C., et al., Space Science Reviews, 86, 1, 1998a.

2. Mewaldt, R. A., R. J Ogliore, G. M. Mason, and G. Gloeckler, "A New Look at Neon-C and SEP-Neon", this volume.

3. Gloeckler, G., et al., Space Science Reviews, 86, 449, 1998.

4. Mason, G. M., et al., Space Science Reviews, 86, 409, 1998.

5. Stone, E. C., et al., Space Science Reviews, 86, 357, 1998b.

6. Stone, E. C., et al., Space Science Reviews, 86, 285, 1998c.

7. von Rosenvinge, T. T., et al., Space Science Reviews, 71, $155,1995$.

8. von Steiger, R., et al. J. Geophys. Research, 105, No. A12, 27217, 2000.

9. Simpson, J. A., Annual. Reviews of Nuclear and Particle Science 33, 706, 1983.

10. Cohen, C. M. S., et al., "Solar Coronal Abundances of Rare Elements Based on Solar Energetic Particles", this volume, 2001. 
11. Gloeckler, G., Fisk, L. A., Zurbuchen, T. H., and Schwadron, N. A., in AIP Conf. Proc. 528, Acceleration and Transport of Energetic Particles Observed in the Heliosphere, ed. R. A. Mewaldt et al., (New York: AIP), 221, 2000.

12. Chotoo, K., et al., J. Geophys. Res., 105, 23107, 2000.

13. Fisk, L. A., Gloeckler, G., Zurbuchen, T. H., and Schwadron, N. A., in AIP Conf. Proc. 528, Acceleration and Transport of Energetic Particles Observed in the
Heliosphere, ed. R. A. Mewaldt et al., (New York: AIP), 229, 2000.

14. Cohen, C. M. S. et al., Geophys. Res. Letters, 26, 2697, 1999.

15. Mason, G. M., et al., Ap. J. 425, L843, 1999.

16. Desai, M. I., et al. "Acceleration of ${ }^{3} \mathrm{He}$ Nuclei at Interplanetary Shocks, Ap.J., 553, L89, 2001. 